\title{
A Vortex Sheet/Point Vortex Dynamical Model for Unsteady Separated Flows
}

\author{
Darwin Darakananda , Jeff D. Eldredge ${ }^{\dagger}$ \\ University of California, Los Angeles, Los Angeles, CA, 90095, USA \\ Tim Colonius $\ddagger$ \\ California Institute of Technology, Pasadena CA 91125, USA \\ David R. Williams $\S$ \\ Illinois Institute of Technology, Chicago IL 60616, USA
}

\begin{abstract}
This paper presents a hybrid vortex sheet/point vortex method for modeling unsteady separated flows. We use vortex sheets to capture the dynamics of the shear layers immediately behind a wing in motion. The sheets provide a natural way of capturing vortex shedding, a feature missing from many point vortex models. We overcome the high computational cost traditionally associated with vortex sheet methods by approximating the spiraling cores of the sheets using point vortices with time-varying circulation. Circulation is continuously truncated from the tips of the vortex sheets and fed into their associated point vortices. To compensate for the discontinuous force response that results from this redistribution of vorticity, we adjust the velocity of the variable strength point vortices. We demonstrate the viability of the method by modeling the impulsive translation of a wing at a fixed angle of attack. We show that the proposed model correctly predicts the dynamics of large-scale vortical structures in the flow by comparing the distribution of vorticity from results of high-fidelity simulation, a model using only vortex sheets, and the proposed model. For the test cases attempted, the hybrid model predicts similar force responses to those of the sheet-only model, while being orders of magnitude faster.
\end{abstract}

\section{Introduction}

The control of separated flow over an airfoil is essential for obtaining lift enhancement, drag reduction, and the overall ability to perform high agility maneuvers. In order to develop reliable flight control systems capable of realizing agile maneuvers, we need a low-order aerodynamics model that can accurately predict the force response of an airfoil to arbitrary disturbances and/or actuation. Unfortunately, classical linearized quasi-steady and impulse response aerodynamic models have difficulty capturing the intrinsically non-linear force response that is observed when we subject the separated flow to a broad spectrum of disturbances or actuation. ${ }^{\mathbb{\nabla}, \mathbb{9}}$ For example, there is experimental evidence showing that lift enhancement from a train of actuator pulses is most effective when the pulse frequency is near or in excess of an inverse convective time unit. ${ }^{\nabla}$ Due to the non-linear interactions of the vortical structures formed in response to these pulses, it is difficult to decompose the total force response into a linear combination of individual pulse responses. Thus, it is natural to consider phenomenological models that can capture this non-linearity in a direct manner.

The early works of Wagner ${ }^{\mathbb{T 7}}$ and Theodorsen ${ }^{\square 6}$ spawned a growing collection of vortex models that aimed to capture the unsteady force response yet still retain an intuitive description of the flow features. Modern versions of these models describe the unsteady dynamics of the wake through the Biot-Savart interactions of vortex elements, ${ }^{\mathbb{t}}$ and some among these have included shedding of vortex elements from the leading edge

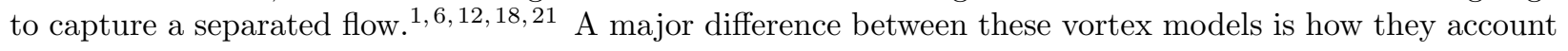

\footnotetext{
*Graduate Student, MAE Dept., email: darwindarak@ucla.edu

†Professor, MAE Dept., email: eldredge@seas.ucla.edu. Associate Fellow AIAA.

¥Professor, MCE Dept., email: colonius@caltech.edu. Associate Fellow AIAA

§Professor, MMAE Dept., email: david.williams@iit.edu. Associate Fellow AIAA
} 
for the shed vorticity. One class of methods model the flux of vorticity into the wake with a routine release of constant-strength vortex elements from one or both edges of the airfoil.[, [1, [6, [1], [4] The models can be based on either discrete vortices or on vortex sheets, which, when discretized, are essentially point vortices whose interconnectivity has been maintained. As the number of vortex elements increases, their interaction with each other results in the emergence of large-scale structures. These methods have been successful in modeling the detailed physics of the development of dynamic stall and the wake, but at the cost of tracking hundreds to thousands of vortices and their interactions. To reduce computational cost, another group of vortex methods directly model the large-scale structures using point vortices with time-varying strength. ㅁ, 피 The flux of vorticity into the fluid is then modeled by dynamically changing the strengths of these point vortices. While this second class of methods is much faster, it is unable to capture the formation of vortical structures from shear layer instabilities.

Neither type of vortex model has been able to accurately predict the force response of an airfoil in flows with finite Reynolds numbers. Recent work by researchers has attempted to address this problem by correcting the models with empirical data. For example, Pitt Ford and Babinsky ${ }^{[3}$ represented a sequence of experimental flow field snapshots as a sequence of potential flows by empirically determining the number of point vortices, as well as their instantaneous positions and strengths. The bound circulation of the airfoil is then adjusted to minimize the difference between the velocity field predicted by the model and that observed empirically. Rather than specifying the positions of the vortices completely using empirical data, Ramesh et al. ${ }^{\text {[I }}$ developed a dynamical vortex model wherein trailing edge vortex shedding is controlled by the Kutta condition, while leading edge vortex shedding is governed by the constraint of the leading edge suction parameter to a certain empirically-determined range. Alternatively, Hemati, Eldredge, and Speyer ${ }^{\text {Th }}$ developed a model optimization framework where existing vortex models are treated as dynamical templates in a constrained optimization problem. While the vortex elements are allowed to convect freely, based on the the variable-strength point vortex model developed by Wang and Eldredge, ${ }^{\mathbb{1 1}}$ their strengths are found by minimizing the difference between model-predicted and empirically measured force.

The objective of this work is to enrich the point vortex template model developed by Wang and Eldredge ${ }^{\text {II }}$ by integrating vortex sheets with point vortices of time-varying strength into a method that is able to capture the formation of coherent vortex structures while remaining computationally tractable for real-time control purposes. This hybrid method requires several considerations, including a new evolution equation for the point vortices to account for the spatial transfer of impulse from the sheet to the vortex, that we address in this paper.

\section{Governing Equations}

The proposed method uses bound vortex sheets to model a moving wing, and a collection of free vortex sheets and point vortices to represent the surrounding wake. To fully specify the dynamics of these vortex elements, we need governing equations for their position and circulation. For notational convenience, we use the two different complex coordinate systems shown in Figure $\mathbf{D}$. We will also denote the regions occupied by the free vortex sheets with $S$ and regions occupied by bound vortex sheets with $S_{b}$.

In classical treatments of this problem, the complex conjugate velocities of all free vortex elements, $w=u-\mathrm{i} v$, are determined by the Biot-Savart integral:

$$
w_{\mathrm{vs}}(z)=\frac{a}{2 \pi \mathrm{i}} \int_{S} \frac{\gamma_{\mathrm{vs}}(s)}{z-z_{\mathrm{vs}}(s)} \mathrm{d} s
$$

for a vortex sheet with sheet strength $\gamma_{\mathrm{vs}}$ distributed along $z_{\mathrm{vs}} \in S$, and

$$
w_{\mathrm{pv}}(z)=\frac{\Gamma_{\mathrm{pv}}}{2 \pi \mathrm{i}} \frac{1}{z-z_{\mathrm{pv}}}
$$

for a point vortex with circulation $\Gamma_{\mathrm{pv}}$ located at $z_{\mathrm{pv}}$. The strength of these free vortex elements are fixed in time to satisfy Kelvin's circulation theorem. Their initial strengths are typically chosen so that the Kutta condition is enforced on the edge from which they are released. The bound vortex sheet on the plate is constrained to follow the same kinematics as the plate itself. Its distribution of vorticity is continuously adjusted to enforce the no-flow-through condition at the plate surface. The proposed method makes two modifications to the classical methods in order to make it tractable for real-time control applications: 


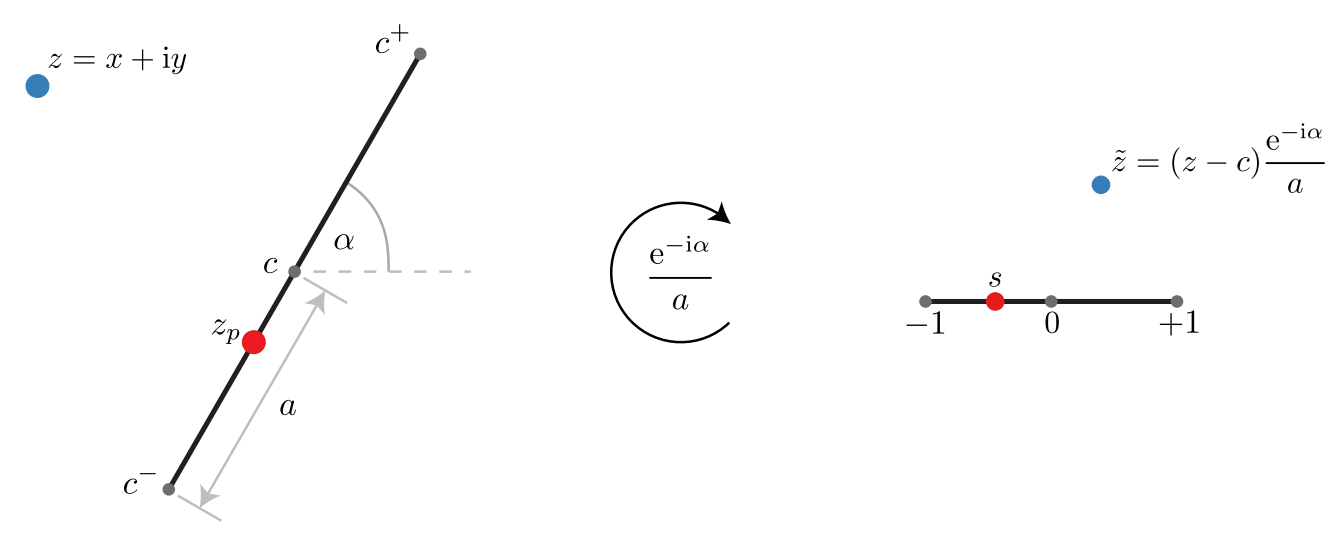

Figure 1. (Left) A fixed frame of reference in which the plate with centroid $c$ and angle of attack $\alpha$ is translating at speed $\dot{c}$ while rotating at rate $\dot{\alpha}$. In this reference frame, location of points in the flow are denoted as $z$ while locations on the plate are denoted as $z_{p}$. The edges of the plate are located at $c^{ \pm}$. (Right) A frame of reference fixed on the centroid of the plate. Points in the flow are denoted as $\tilde{z}$ while points on the plate are denoted as a scalar $s$. The lengths are scaled so that the leading and trailing edges of the plate correspond to $s=1$ and $s=-1$, respectively.

- Inspired by the work in Ref. [2, we allow for the use of a suction parameter on each plate edge to determine the flux of vorticity into the flow. By allowing the vortex sheet strength to be singular at the edges of the plate, we remove the model closure that is typically provided by the Kutta condition. This gives us the option of tuning the suction parameters using empirical data. (We also retain the ability to enforce the Kutta condition at either or both edges by setting their respective suction parameters to zero.)

- We approximate the spiral cores of vortex sheets using point vortices with time-varying strength. The vortex sheets, due to their increase in complexity over time, are the most computationally taxing component in these methods. By continuously feeding parts of the sheet into point vortices, we can restrict the growth of computational cost. The governing equation of the variable strength point vortex is based on a modified form of the impulse matching equation developed in Ref. 18.

The governing equations corresponding to these changes are described in the following sections. Details on some of the equations' derivations are included in the Appendix.

\section{A. Bound Vortex Sheet}

The role of the bound vortex sheet is to enforce the no-flow-through condition on the plate. The distribution of vorticity along the bound sheet, $\gamma_{\mathrm{bs}}$, must induce a velocity field that cancels out all normal velocities along the plate, $V_{\perp}$ :

$$
\operatorname{Im}\left\{\mathrm{e}^{-\mathrm{i} \alpha} \frac{a}{2 \pi \mathrm{i}} \int_{S_{b}} \frac{\gamma_{\mathrm{bs}}(s)}{z-z_{\mathrm{bs}}(s)} \mathrm{d} s\right\}+V_{\perp}(s)=0 \quad z \in S_{p} .
$$

Traditionally, the solution to the equation above is found by decomposing $V_{\perp}$ into its Fourier series. This in turn gives a Fourier expansion for $\gamma_{\mathrm{bs}}$ that assumes that the Kutta condition is enforced at one or both of the edges. Since we want the option of empirically determining the flux of vorticity from the plate into the flow, we cannot assume that the Kutta condition is satisfied. Instead, following the steps outlined by Jones, ${ }^{1,}$ we decompose $V_{\perp}$ as a Chebyshev series:

$$
V_{\perp}(s)=\sum_{n=0}^{\infty} C_{n} T_{n}(s) \quad s \in[-1,1],
$$

where the $T_{n}$ 's are Chebyshev polynomials of the first kind. It can then be shown that in order to satisfy conservation of global circulation and equation (31), the bound vortex sheet strength must take the form

$$
\gamma_{\mathrm{bs}}(s)=-\frac{1}{\sqrt{1-s^{2}}}\left[2\left(C_{0}+\operatorname{Im}\left\{\dot{c} \mathrm{e}^{-\mathrm{i} \alpha}\right\}\right) s+C_{1}+\dot{\alpha} a\left(2 s^{2}-1\right)+\frac{1}{\pi} \int_{A_{f}} \mathrm{~d} \Gamma\right]-2 \sum_{n=1}^{\infty} C_{n} \sqrt{1-s^{2}} U_{n-1}(s),
$$


where $U_{n}$ are Chebshev polynomials of the second kind. Unlike the classical formulation, the equation above allows the vortex sheet strength to be infinite at both edges of the plate. The term in square brackets, evaluated at the leading and trailing edges, are denoted as $\mathrm{SP}_{+}$and $\mathrm{SP}_{-}$, respectively:

$$
\mathrm{SP}_{ \pm}:=C_{1}+\dot{\alpha} a+\frac{1}{\pi} \int_{A_{f}} \mathrm{~d} \Gamma \pm 2\left(C_{0}+\operatorname{Im}\left\{\dot{\mathrm{c}} \mathrm{e}^{-\mathrm{i} \alpha}\right\}\right) .
$$

Following the same steps taken in Ref. II2, it can be shown that $\mathrm{SP}_{ \pm}$are measures of suction at the edges of the plate. For an infinitely thin plate, an edge with nonzero suction implies that the velocity at that edge is infinite. This singular behavior is only a consequence of modeling a real airfoil with an infinitely thin plate, as real airfoils with rounded edges can support finite suction before flow separation occurs. ${ }^{\text {耳 }}$ In the point vortex model introduced in Ref. [2, the support for leading edge suction is modeled by shedding vorticity into the wake at a rate such that the Kutta condition is enforced at the trailing edge, and $\mathrm{SP}_{+}$is constrained to be less than a critical leading edge suction parameter determined from high-fidelity simulation results. In a similar manner, the proposed model can treat the suction at both edges, $\mathrm{SP}_{ \pm}$, as parameters that can be tuned against empirical or simulation results. In this work, we will set it to zero at both edges, consistent with the Kutta condition.

Rather than treating the bound vortex sheet of the plate as a single entity, it will be useful to consider it as a superposition of multiple sheets

$$
\gamma_{\mathrm{bs}}=\sum_{i=1}^{N} \gamma_{i}+\gamma_{m}
$$

where

- $N$ is the number of free vortex elements,

- $\gamma_{i}$ is the bound sheet created in response to the velocity induced by the $i$-th vortex element in the fluid,

- $\gamma_{m}$ is the sheet responsible for accounting for the normal velocity of by the plate's motion.

Denoting $V_{\perp}^{i}$ as the portion of $V_{\perp}$ induced by the $i$-th vortex element, we obtain a new set of Chebyshev coefficients

$$
V_{\perp}^{i}(s)=\sum_{n=0}^{\infty} C_{n}^{i} T_{n}(s) \quad s \in[-1,1] .
$$

The distribution of vorticity in the individual sheets can then be written as

$$
\gamma_{i}(s)=-\frac{1}{\sqrt{1-s^{2}}}\left[2 C_{0}^{i} s+C_{1}^{i}+\frac{1}{\pi} \int_{A_{i}} \mathrm{~d} \Gamma\right]-2 \sum_{n=1}^{\infty} C_{n}^{i} \sqrt{1-s^{2}} U_{n-1}(s)
$$

for sheets corresponding to free vortex elements, and

$$
\gamma_{m}(s)=-\frac{1}{\sqrt{1-s^{2}}}\left[2 \operatorname{Im}\left\{\mathrm{e}^{-\mathrm{i} \alpha} \dot{c}\right\} s+\dot{\alpha} a\left(2 s^{2}-1\right)\right]
$$

for the sheet corresponding to plate motion. Equations (G) and (G) will be used to derive expressions for the force response.

\section{B. Aerodynamic Force}

The vector force on an infinity-thin flat plate, $\boldsymbol{F}$, can be expressed in terms of linear impulse in the fluid, $\boldsymbol{P}$, through the relation

$$
\boldsymbol{F}=-\rho_{f} \frac{\mathrm{d} \boldsymbol{P}}{\mathrm{d} t}
$$

where $\rho_{f}$ is the density of the fluid., [3, 20] The impulse can be linearly decomposed into the impulse of the free vortex elements and their corresponding bound vortex sheet, $\boldsymbol{P}_{f}$, and the impulse of the bound vortex sheet created in response to rigid body motion, $\boldsymbol{P}_{m}$ :

$$
\boldsymbol{P}=\boldsymbol{P}_{f}+\boldsymbol{P}_{m}
$$


with

$$
\begin{aligned}
& \boldsymbol{P}_{f}=\sum_{i=1}^{N} \boldsymbol{P}_{f_{i}}=\sum_{i=1}^{N}[\underbrace{\int_{A_{i}} \boldsymbol{x} \times \boldsymbol{\omega} \mathrm{d} A}_{\begin{array}{c}
\text { impulse of } \\
\text { vortex element }
\end{array}}+\underbrace{\int_{S_{p}} \boldsymbol{x} \times \boldsymbol{\gamma}_{i} \mathrm{~d} s}_{\begin{array}{c}
\text { impulse of } \\
\text { responding bound } \\
\text { vortex sheet }
\end{array}}] \\
& \boldsymbol{P}_{m}=\int_{S_{p}} \boldsymbol{x} \times \gamma_{\boldsymbol{m}} \mathrm{d} s .
\end{aligned}
$$

Using Eqs. (ШШ) and (घ), we find the complex form of $\boldsymbol{P}_{m}$ :

$$
P_{m}=-\mathrm{i} \int_{S_{b}} z \gamma_{m} \mathrm{~d} s=\mathrm{i} \pi \mathrm{e}^{\mathrm{i} \alpha} \operatorname{Im}\left\{\mathrm{e}^{-\mathrm{i} \alpha} \dot{c}\right\} .
$$

The explicit form for $\boldsymbol{P}_{f_{i}}$ depends on the specific type of vortex element we are interested in. After combining

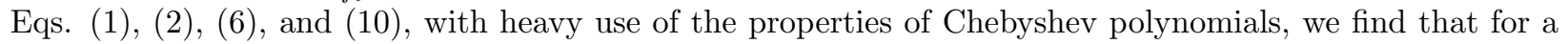
free vortex sheet, the complex form of $\boldsymbol{P}_{f}$ is

$$
P_{f, v s}=-\mathrm{ie}^{\mathrm{i} \alpha} \int_{S} p\left(z_{s}\right) \mathrm{d} \Gamma
$$

and for a single point vortex at $z_{\mathrm{pv}}$ it is

$$
P_{f, p v}=-\mathrm{ie}^{\mathrm{i} \alpha} \Gamma_{\mathrm{pv}} p\left(z_{\mathrm{pv}}\right),
$$

where

$$
p(z)=\operatorname{Re}\{[\tilde{z}(z)-\sqrt{\tilde{z}(z)-1} \sqrt{\tilde{z}(z)+1}]\}-\tilde{z}(z),
$$

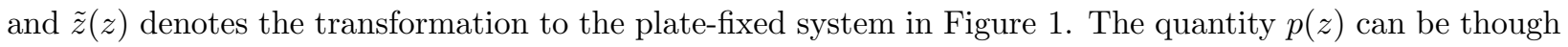
of as the impulse per unit circulation for a free vortex element adjacent to a plate. The net impulse in the fluid can then be written as

$$
P=\mathrm{ie}^{\mathrm{i} \alpha}\left(\tilde{P}_{\mathrm{m}}+\tilde{P}_{\mathrm{pv}}+\tilde{P}_{\mathrm{vs}}\right)=\mathrm{ie}^{\mathrm{i} \alpha}[\underbrace{\pi \operatorname{Im}\left\{\mathrm{e}^{-\mathrm{i} \alpha} \dot{c}\right\}}_{\text {motion }}+\underbrace{\sum_{v=1}^{N_{v}} \Gamma_{v} p\left(z_{v}\right)}_{\text {point vortex }}+\underbrace{\int_{S} p\left(z_{s}\right) \mathrm{d} \Gamma_{s}}_{\text {vortex sheet }}] .
$$

\section{Transfer of Circulation}

As the vortex sheets grow behind the plate, the computational cost for the vortex-to-vortex interactions increases. In the best case, the cost increases linearly with time. Practically, however, as the sheets start to roll up and stretch, the growth in complexity can become exponential. Since we want to use this model for real-time flow control applications, we need a way to keep the computational cost approximately constant in time. We propose to model the spiral core of each vortex sheet as a point vortex with time-varying strength. Instead of waiting for the vorticity in the sheet to asymptotically converge on its spiral core, we continuously truncate the sheet and directly feed vorticity into a point vortex. The rate at which we transfer the vorticity, $\dot{\Gamma}$, is allowed to be variable, as it could provide an additional degree of freedom we can use to tune the model against empirical data. The redistribution of vorticity changes the impulse of both the vortex sheet and the point vortex. In general, because of the transfer of circulation across space - from the sheet to the point vortex - the change in the vortex sheet's impulse resulting from this transfer is not equal and opposite to the change in the point vortex's impulse. This implies, via Eqs. (ㅈ) and (미), that simply transferring the circulation without considerations for impulse can result in discontinuous changes in the force on the

plate. We can compensate for this discontinuity by modifying the state of the point vortex so that the total impulse of the system, as well as its rate of change, is preserved during the transfer process.

As shown above, the impulse and rate of change of impulse of a point vortex depends on its position, circulation, velocity, and rate of change of circulation

$$
\begin{aligned}
& P_{\mathrm{pv}}=P_{\mathrm{vs}}\left(\Gamma_{\mathrm{pv}}, z_{\mathrm{pv}}\right), \\
& \dot{P}_{\mathrm{pv}}=\dot{P}_{\mathrm{pv}}\left(\Gamma_{\mathrm{pv}}, z_{\mathrm{pv}}, \dot{\Gamma}_{\mathrm{pv}}, \dot{z}_{\mathrm{pv}}\right) .
\end{aligned}
$$


Since changing the instantaneous position and strength of the point vortex changes its impulse, and its rate of change of strength must match the sheet truncation rate (i.e. the negative of the rate of change of the circulation of the sheet), the only parameter still available for us to change is the point vortex's velocity. A constant-strength point vortex moves with the so-called Kirchhoff velocity, $w^{\star}\left(z_{\mathrm{pv}}\right)$, due to the contributions induced by other vortices and by the body motion and free stream. To this we add a correction term, $\Delta \dot{z}_{\mathrm{pv}}$, to obtain the adjusted motion of the variable-strength point vortex:

$$
\dot{z}_{\mathrm{pv}}=w^{\star}\left(z_{\mathrm{pv}}\right)+\Delta \dot{z}_{\mathrm{pv}} .
$$

In Appendix subsection $\mathbb{A}$, we show that the appropriate velocity correction, to account for the mismatch in impulse due to the transfer of circulation, can be written as

$$
\Delta \dot{z}=a \mathrm{e}^{\mathrm{i} \alpha} \frac{\dot{\Gamma}}{\Gamma}\left[\frac{\left(p_{\mathrm{tip}}-p_{\mathrm{pv}}\right)\left(1+\eta^{\star}\right)+\left(p_{\mathrm{tip}}^{\star}-p_{\mathrm{pv}}^{\star}\right)\left(1-\eta^{\star}\right)}{\eta+\eta^{\star}} .\right]
$$

where

$$
\eta:=-\frac{z_{\mathrm{pv}}-c}{\sqrt{\left(z_{\mathrm{pv}}-c^{+}\right)\left(z_{\mathrm{pv}}-c^{-}\right)}}
$$

and $p_{\mathrm{pv}}$ and $p_{\text {tip }}$ are the impulse per unit circulation of the point vortex and the tip of the vortex sheet, respectively. Equation (ㅍ) represents an extension of the impulse matching model developed by Wang and Eldredge, ${ }^{\text {IX }}$ which accounted for vorticity flux from the plate directly into the variable-strength point vortex, to a more general model that accounts for transfer of vorticity from one vorticity element to another.

\section{Results}

In this section, we evaluate the viability of the proposed model on problem involving the impulsive translation of a flat plate at a fixed angle of attack of 60 degrees. We will compare the model with a model in which only a vortex sheet is used at each edge and with a Navier-Stokes solution of the same problem, using high-fidelity CFD, at Reynolds number 1000. In the current test problem, we do not yet incorporate empirical data into our model, leaving that for future work. The flux of circulation from the plate into the flow is simply governed by the Kutta condition on both ends, with $\mathrm{SP}_{ \pm}=0$. The remaining details of the numerical solution are described in the Appendix subsection $\mathbb{B}$.

Figure $\nabla$ compares the flow structures near the plate at various convective times. We see that the inviscid physics used by the vortex models were able to predict vortical structures that closely match the vorticity distribution obtained from the high-fidelity viscous simulation. Both the sheet-only model and the hybrid model capture the roll-up of a new trailing edge vortex from an instability in the shear layer. Figure [1] shows the predicted force and number of control points used for a particular schedule of circulation transfer rates between the sheets and the point vortices. While we eventually plan on determining appropriate transfer rates using empirical data, the transfer schedule in Figure 3 was picked manually. Without the impulse matching velocity correction, the predicted force is quite different from the force predicted by the vortex sheet only model. Furthermore, it is discontinuous whenever the rate of circulation transfer changes. Restricting the rate of circulation transfer to be constant would make it difficult to handle vortex shedding events, since the flux of vorticity from the sheet to the old point vortex is not necessarily the same as the flux into a newly-developing point vortex.

Once the velocity of the point vortex is adjusted with equation ([च), the instantaneous force becomes insensitive to discontinuities in the circulation transfer rate, as apparent in Figure 3. Furthermore, when a new roll-up occurs on the sheet, a new point vortex can be released without disrupting the predicted force (see bottom right plot in Figure [2). The force predicted by the hybrid model agrees quite well with the sheet-only model for most of the interval shown in Figure B. Moreover, the force response from both the sheet-only model and the proposed hybrid model show good qualitative agreement with results from high-fidelity simulation. The computational cost of the hybrid model is orders of magnitude smaller than that of the vortex sheet model (as shown in the middle panel in Figure B) and several more orders smaller than the high-fidelity CFD simulation. More importantly, the cost of the hybrid model remains relatively constant as the flow develops, since the vortex sheets are not allowed to grow indefinitely.

Figure 1 shows the force predicted by the hybrid model with different rates of circulation transfer. The variations in the force response suggests that the model can be tuned against empirical data by adjusting 
$U t /(2 a)=1.5$

$U t /(2 a)=2.5$

$U t /(2 a)=3.5$
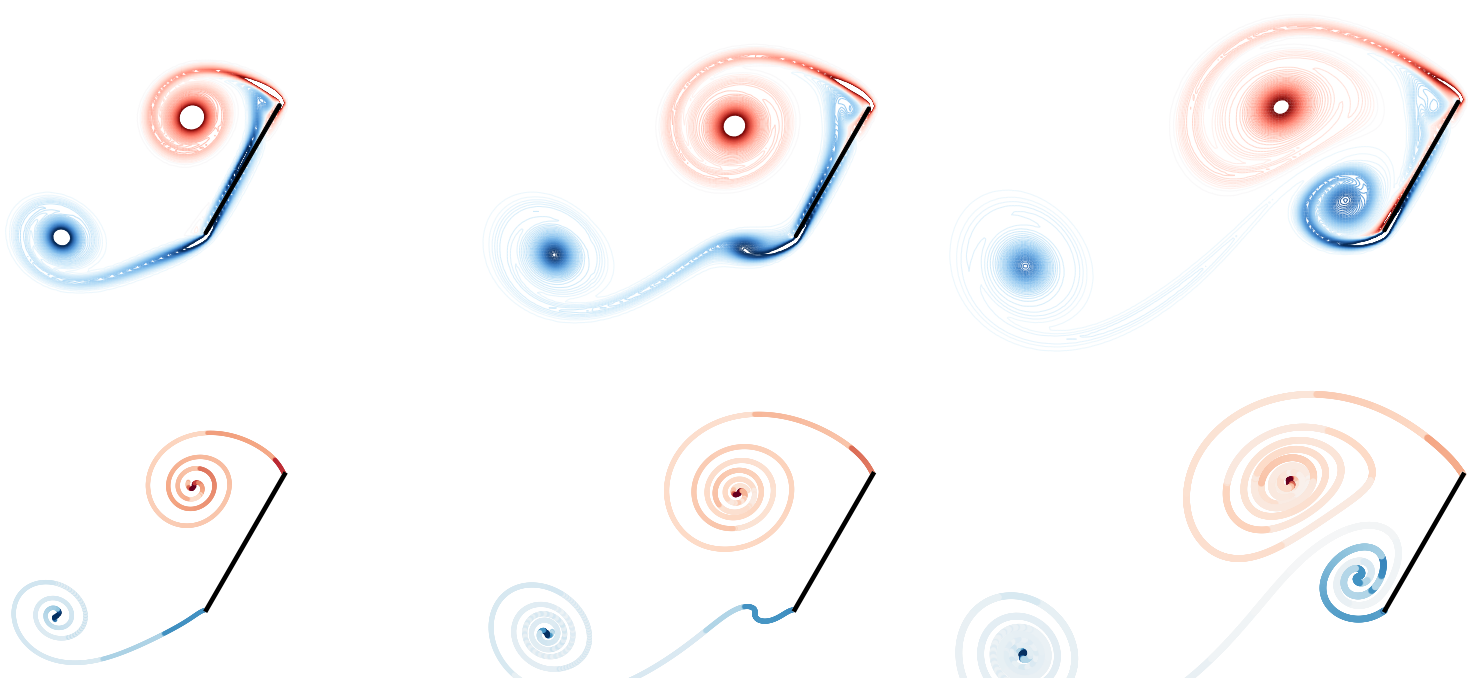

$N=1144$
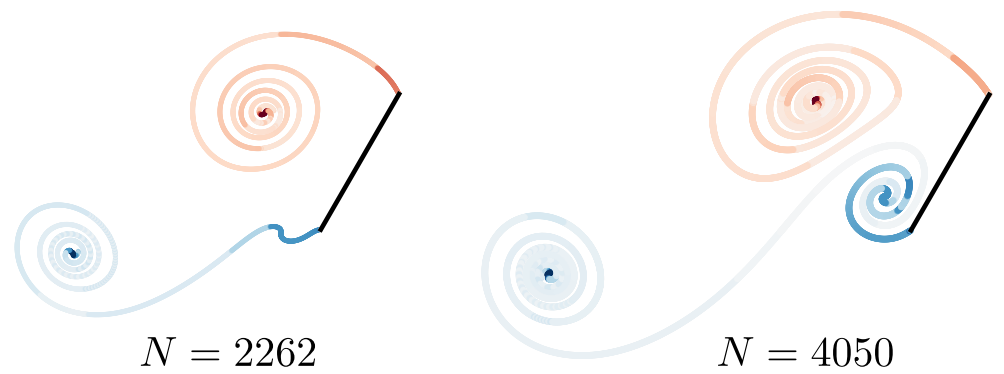

$N=2262$

$N=4050$

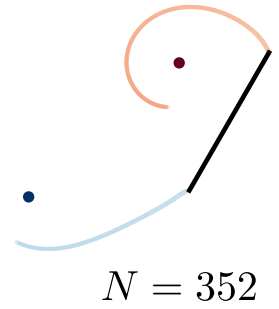

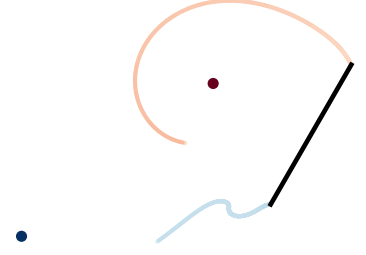

$N=516$

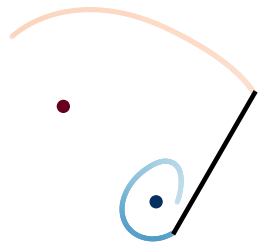

$N=473$

Figure 2. Comparison of the predicted large scale structures around the plate using high fidelity CFD at Re $=1000$ (top row), a vortex sheet model (middle row), and the proposed hybrid point/sheet model (bottom row). The CFD results show the vorticity contours. The vortex models show the strength and positions of the vortex sheets and point vortices, as well as the number of control points used to resolve the vortex sheet.

the circulation transfer rate via an optimization procedure similar to Ref. H. However, regardless of the circulation transfer rate, all the inviscid models predict an earlier trailing-edge vortex shedding event than the viscous simulation results.

\section{Conclusion}

In this work, we demonstrated the viability of a hybrid vortex sheet/point vortex method in modeling the force response of unsteady separated flows. We use vortex sheets to capture the development of coherent vortices from instabilities in the shear layer. To avoid the high computational cost traditionally associated with vortex sheet methods, we use point vortices with time-varying strength as an approximation of the vortex sheet's spiral cores. By siphoning circulation from the ends of the vortex sheets into point vortices, we can keep the vortex sheets short and fast to work with. A modification to the classical governing equations of a point vortex allows instantaneous transfer of circulation from vortex sheets to point vortices without adversely affecting the predicted force.

Instead of pursuing a closed model, we have kept a number of parameters open to adjustment. By varying the rate of circulation transfer between vortex sheets and point vortices, we have some degree of control over the distribution of vorticity in the flow. While the instantaneous force is insensitive to the transfer rate by construction, the redistribution of vorticity can be used to shape the long term force response. Additionally, the Chebyshev series formulation of the bound vortex sheet strength exposes the suction parameters, thus 

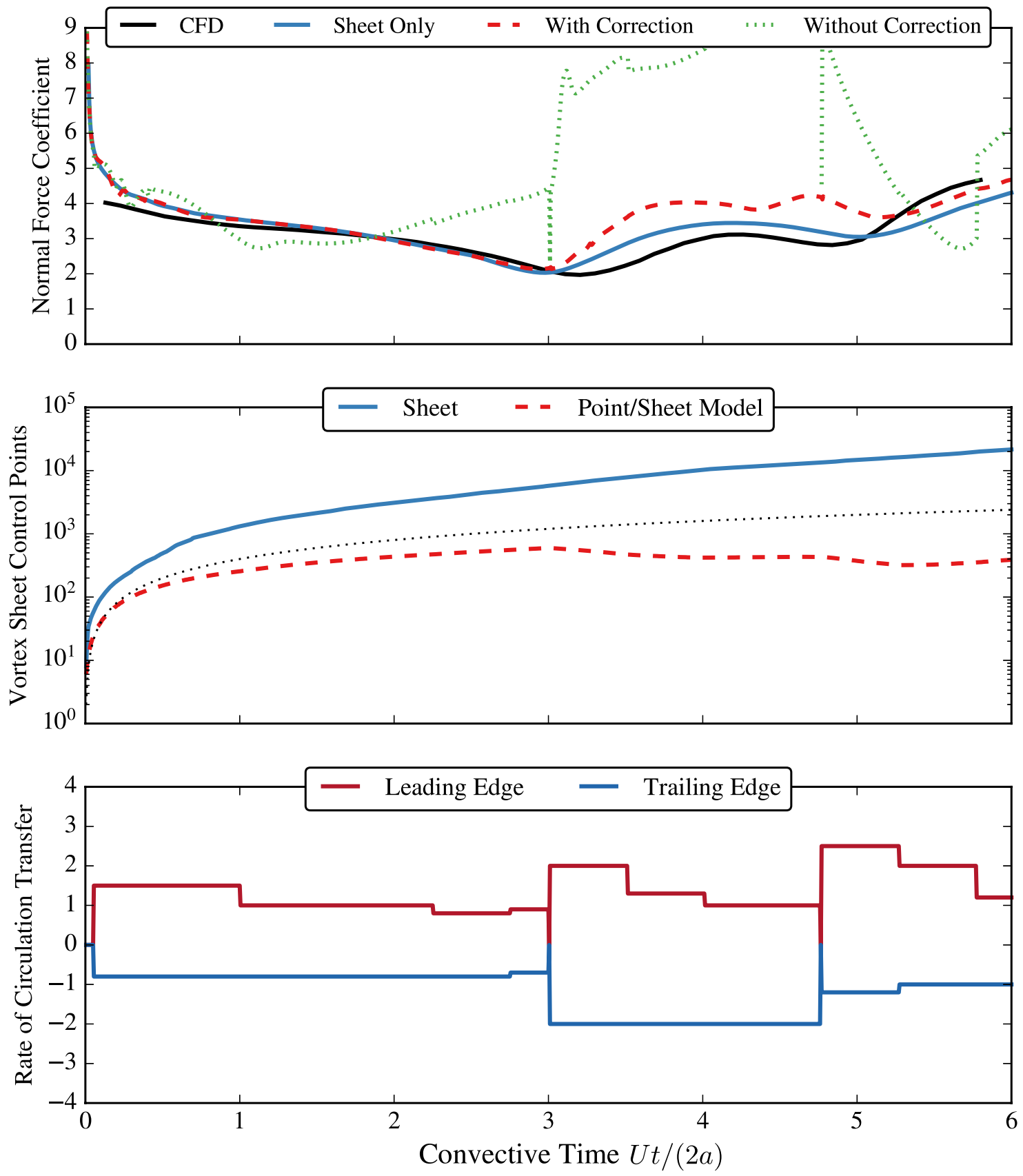

Figure 3. (Top) A comparison of the force response predicted by high fidelity CFD, a vortex sheet model, a hybrid model where we do not correct for the change in impulse, and a hybrid model with the appropriate velocity corrections. (Middle) The dotted black line shows the number of computational element over time if only a single vortex sheet segment is released from each edge per time step. The solid blue line represents the case where additional points are inserted between segments to maintain connectivity in the sheet. The solid red line corresponds to the proposed method. (Bottom) The red and blue lines show the rate at which circulation is transfered from the vortex sheets to their associated point vortex at the leading and trailing edges, respectively.

giving us a straightforward way of controlling the vorticity flux from the plate into the vortex sheets, as in the work by Ramesh et al. ${ }^{\text {W }}$ This set of tunable parameters opens up the possibility of using the method as a dynamical template in a data-driven framework..$^{-1}$ In future work, we intend to incorporate empirical data (e.g. pressure/force measurements on a wing) as a means of recovering some of the flow physics that were 


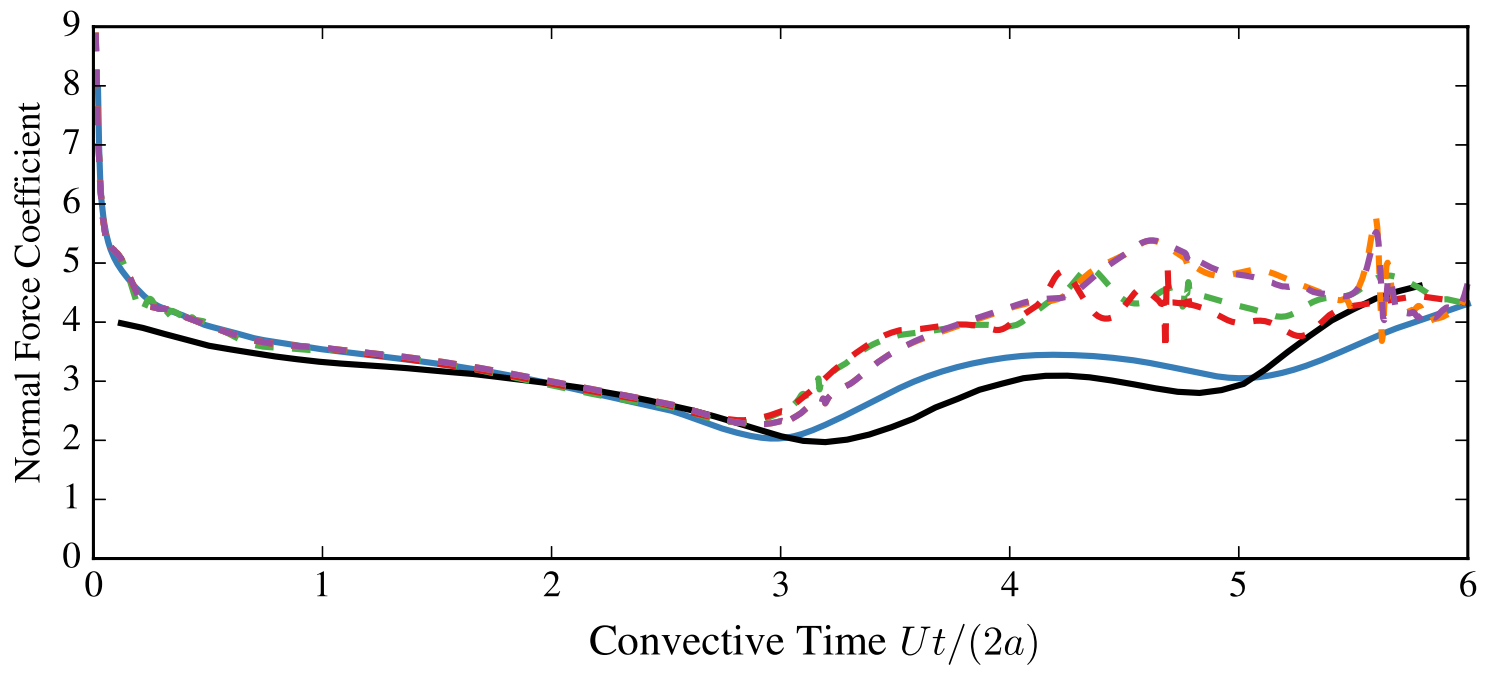

Figure 4. The comparison between the force predicted by high fidelity CFD (solid black), a sheet-only model (solid blue), and the hybrid model with different rates of circulation transfer (dashed lines).

discarded for the sake of simplicity and speed.

\section{Appendix}

\section{A. Derivation of Velocity Correction}

We want the instantaneous force on the plate to be unaffected by the rate at which we transfer circulation from the vortex sheet to the point vortex. Let superscript $\dagger$ indicate properties for which there is transfer of circulation. Since the force can be expressed in terms of the rate of change of linear impulse, we require that

$$
\frac{\mathrm{d} P^{\dagger}}{\mathrm{d} t}(t)=\frac{\mathrm{d} P}{\mathrm{~d} t}(t)
$$

The rate of change of impulse can be decomposed into contributions from the plate and all the free vortex elements:

$$
\frac{\mathrm{d} P}{\mathrm{~d} t}=-\dot{\alpha} \mathrm{e}^{\mathrm{i} \alpha}\left(\tilde{P}_{\mathrm{m}}+\tilde{P}_{\mathrm{pv}}+\tilde{P}_{\mathrm{vs}}\right)+\mathrm{e}^{\mathrm{i} \alpha}\left(\frac{\mathrm{d} \tilde{P}_{\mathrm{m}}}{\mathrm{d} t}+\frac{\mathrm{d} \tilde{P}_{\mathrm{pv}}}{\mathrm{d} t}+\frac{\mathrm{d} \tilde{P}_{\mathrm{vs}}}{\mathrm{d} t}\right) .
$$

Since instantaneous impulse and the plate kinematics are unaffected by the rate of circulation transfer, Eqs. (ㅁ. $)$ and (ख्य) can be combined into

$$
\frac{\mathrm{d} \tilde{P}_{\mathrm{vs}}^{\dagger}}{\mathrm{d} t}(t)-\frac{\mathrm{d} \tilde{P}_{\mathrm{vs}}}{\mathrm{d} t}(t)=\frac{\mathrm{d} \tilde{P}_{\mathrm{pv}}}{\mathrm{d} t}(t)-\frac{\mathrm{d} \tilde{P}_{\mathrm{pv}}^{\dagger}}{\mathrm{d} t}(t)
$$

The impulse from the vortex sheet can be split into the part that will be truncated and the part that will remain:

$$
\tilde{P}_{\mathrm{vs}}^{\dagger}(t)=\tilde{P}_{\mathrm{vs}}(t)=\underbrace{\int_{0}^{\Gamma_{-}} p(z) \mathrm{d} \Gamma}_{\begin{array}{c}
\text { part to be } \\
\text { truncated }
\end{array}}+\underbrace{\int_{\Gamma_{-}}^{\Gamma_{+}} p(z) \mathrm{d} \Gamma}_{\text {part to keep }}
$$

The impulse of the vortex sheet after a small $\Delta t$ can be written as

$$
\tilde{P}_{\mathrm{vs}}(t+\Delta t)=\tilde{P}_{\mathrm{vs}}(t)+\frac{\mathrm{d}}{\mathrm{d} t}\left(\int_{0}^{\dot{\Gamma} \Delta t} p(z) \mathrm{d} \Gamma\right) \Delta t+\frac{\mathrm{d}}{\mathrm{d} t}\left(\int_{\dot{\Gamma} \Delta t}^{\Gamma_{+}} p(z) \mathrm{d} \Gamma\right) \Delta t+O\left(\Delta t^{2}\right)
$$


when there is no transfer of circulation, and

$$
\tilde{P}_{\mathrm{vs}}^{\dagger}(t+\Delta t)=\tilde{P}_{\mathrm{vs}}(t)-\left(\int_{0}^{\dot{\Gamma} \Delta t} p(z) \mathrm{d} \Gamma\right) \Delta t+\frac{\mathrm{d}}{\mathrm{d} t}\left(\int_{\dot{\Gamma} \Delta t}^{\Gamma_{+}} p(z) \mathrm{d} \Gamma\right) \Delta t+O\left(\Delta t^{2}\right)
$$

when we transfer circulation at the rate $\dot{\Gamma}=\frac{\Gamma_{-}}{\Delta t}$. The difference in the rate of change of impulse for the vortex sheet is

$$
\begin{aligned}
\frac{\mathrm{d} \tilde{P}_{\mathrm{vs}}^{\dagger}}{\mathrm{d} t}-\frac{\mathrm{d} \tilde{P}_{\mathrm{vs}}}{\mathrm{d} t} & =-\lim _{\Delta t \rightarrow 0}\left(\frac{\int_{0}^{\dot{\Gamma} \Delta t} p(z) \mathrm{d} \Gamma}{\Delta}+\frac{\mathrm{d}}{\mathrm{d} t} \int_{0}^{\dot{\Gamma} \Delta t} p(z) \mathrm{d} \Gamma\right) \\
& =-\dot{\Gamma} p_{\text {tip }}
\end{aligned}
$$

where $p_{\text {tip }}$ is the impulse per unit circulation at the end of the vortex sheet. Combining the equation above and Eq. (四), and substituting Eq. ([4]) for the impulse of a point vortex, we find

$$
\frac{\mathrm{d} p^{\dagger}}{\mathrm{d} t}\left(z_{\mathrm{pv}}\right)-\frac{\mathrm{d} p}{\mathrm{~d} t}\left(z_{\mathrm{pv}}\right)=\frac{\dot{\Gamma}}{\Gamma_{\mathrm{pv}}}\left[p\left(z_{\mathrm{pv}}\right)-p\left(z_{\mathrm{tip}}\right)\right]
$$

which can be expanded into the velocity correction term stated in Eqs. (미) and ([ष]).

\section{B. Implementation Details}

The vortex sheets are divided into multiple segments with a series of control points. Integrals over a free vortex sheet is approximated using the trapezoid rule:

$$
\int f(\Gamma) \mathrm{d} \Gamma \approx \sum_{i=1}^{N} f\left(\Gamma_{i}\right) \Delta \Gamma_{i}
$$

where the $i$ indexes over all the control points, and

$$
\Delta \Gamma_{i}:= \begin{cases}\frac{1}{2}\left(\Gamma_{2}-\Gamma_{1}\right) & i=1 \\ \frac{1}{2}\left(\Gamma_{i+1}-\Gamma_{i-}\right) & 1<i<N \\ \frac{1}{2}\left(\Gamma_{N}-\Gamma_{N-1}\right) & i=N .\end{cases}
$$

As in Refs. 9, [0, we compute the self-induced velocity of a free vortex sheet with a blob kernel instead of the usual Cauchy kernel in order to suppress numerical instabilities :

$$
\begin{aligned}
w_{\mathrm{vs}}(z) & =\frac{1}{2 \pi \mathrm{i}} \int\left(\frac{\left|z-z_{\mathrm{vs}}\right|^{2}}{\left|z-z_{\mathrm{vs}}\right|^{2}+\delta^{2}}\right) \frac{1}{z-z_{\mathrm{vs}}} \mathrm{d} \Gamma \quad \text { for } z \in S \\
& \approx \sum_{i=1}^{N}\left(\frac{\left|z-z_{i}\right|^{2}}{\left|z-z_{i}\right|^{2}+\delta^{2}}\right) \frac{\Delta \Gamma_{i}}{z-z_{i}}
\end{aligned}
$$

where $\delta$ is a smoothing radius.

For the bound vortex sheets, its $N_{p}$ control points are distributed along the plate as a Lobatto grid:

$$
s_{i}=\cos \left(\frac{(n-1) \pi}{N-1}\right) \quad n \in 1, \cdots, N_{p}
$$

where the density of points is higher near the edges of sheet. This distribution of points gives us a straightforward way to approximate the coefficients in Eq. (国) using the discrete Chebyshev transform. These approximate coefficients can then be used to compute a truncated series solution for the sheet's strength

$$
\gamma_{i}(s) \approx-\frac{1}{\sqrt{1-s^{2}}}\left[2 C_{0}^{i} s+C_{1}^{i}+\frac{1}{\pi} \int_{A_{i}} \mathrm{~d} \Gamma\right]-2 \sum_{n=1}^{N_{p}} C_{n}^{i} \sqrt{1-s^{2}} U_{n-1}(s) .
$$


Using the exact solutions to relevant integrals of Chebyshev polynomials described in Ref. ㅍ.5, we find that the velocity induced by all the bound vortex sheets as

$$
w_{=}(z)= \begin{cases}-\mathrm{ie}^{-\mathrm{i} \alpha} \sum_{n=0}^{\infty} C_{n} T_{n}(\tilde{z}) & : z \in S_{p} \\ \mathrm{ie}^{-\mathrm{i} \alpha}\left[\frac{\sum \Gamma_{\omega} / \pi+C_{1}+2 C_{0} \tilde{z}}{2 \sqrt{\tilde{z}-1} \sqrt{\tilde{z}+1}}-\sum_{n=0}^{\infty} C_{n} J_{+}^{-1}(\tilde{z})^{n}\right] & : \text { otherwise. }\end{cases}
$$

\section{Shedding New Vortices}

The discretized form of the impulse of a vortex sheet can be written as

$$
P_{\mathrm{vs}} \approx \mathrm{ie}^{\mathrm{i} \alpha} \sum_{i=1}^{N_{s}} p\left(z_{i} ;, c, \alpha\right) \Delta \Gamma_{i}=\mathrm{ie}^{\mathrm{i} \alpha}\left[p\left(z_{1} ; c, \alpha\right) \Delta \Gamma_{1}+\sum_{i=2}^{N_{s}} p\left(z_{i} ;, c, \alpha\right) \Delta \Gamma_{i}\right] .
$$

The first term in the square brackets is already in the from of the impulse of a point vortex. We can release a new point vortex without affecting the impulse of the system by simply treating the last segment of the discretized vortex sheet as a point vortex.

\section{Acknowledgments}

Support by the U.S. Air Force Office of Scientific Research (FA9550-14-1-0328) with program manager Dr. Douglas Smith is gratefully acknowledged.

\section{References}

${ }^{1}$ S. A. Ansari, R. Zbikowski, and K. Knowles. Non-linear unsteady aerodynamic model for insect-like flapping wings in the hover. Part 1: methodology and analysis. Proc. IMechE, Part G: J. Aerospace Engineering, 220:61-83, 2006.

${ }^{2}$ J. D. Eldredge. A Reconciliation of Viscous and Inviscid Approaches to Computing Locomotion of Deforming Bodies. Exp. Mech., 50(9):1349-1353, July 2009.

${ }^{3}$ C. W. Pitt Ford and H. Babinsky. Lift and the leading-edge vortex. J. Fluid Mech., 720:280-313, 2013.

${ }^{4}$ M. S. Hemati, J. D. Eldredge, and J. L. Speyer. Improving vortex models via optimal control theory. J. Fluid Struct, 49:91-111, 2014

${ }^{5}$ M. A. Jones. The separated flow of an inviscid fluid around a moving flat plate. J. Fluid Mech., 496:405-441, December 2003.

${ }^{6}$ J. Katz. A discrete vortex method for the non-steady separated flow over an airfoil. J. Fluid Mech., 102, 1981.

${ }^{7}$ J. Katz and A. Plotkin. Low-Speed Aerodynamics. Cambridge University Press, 2nd edition, 2001.

${ }^{8}$ W. Kerstens, D. Williams, J. Pfeiffer, R. King, and T. Colonius. Closed loop control of a wing's lift for 'gust' suppression. 5th AIAA Flow Control Conference, June 2010, Chicago. AIAA Paper 2010-4969, 2010.

${ }^{9}$ R. Krasny. Computation of vortex sheet roll-up in the Trefftz plane. J. Fluid Mech., 184:123-155, 1987.

${ }^{10}$ Robert Krasny. A study of singularity formation in a vortex sheet by the point-vortex approximation. J. Fluid Mech., 167(-1):65, 1986.

${ }^{11}$ S. Michelin and S. G. Llewellyn Smith. An unsteady point vortex method for coupled fluid-solid problems. Theor. Comput. Fluid Dyn., 23(2):127-153, May 2009.

${ }^{12}$ Kiran Ramesh, Ashok Gopalarathnam, Kenneth Granlund, Michael V. Ol, and Jack R. Edwards. Discrete-vortex method with novel shedding criterion for unsteady aerofoil flows with intermittent leading-edge vortex shedding. J. Fluid Mech., 751:500$538,2014$.

${ }^{13}$ P. G. Saffman. Vortex dynamics. Cambridge university press, 1992.

${ }^{14}$ R. K. Shukla and J. D. Eldredge. An inviscid model for vortex shedding from a deforming body. Theor. Comput. Fluid Dyn., 21(5):343-368, July 2007.

${ }^{15}$ R. M. Slevinsky and S. Olver. A fast and well-conditioned spectral method for singular integral equations. arXiv:1507.00596, July 2015.

${ }^{16} \mathrm{~T}$. Theodorsen. General theory of aerodynamic instability and the mechanism of flutter. Technical Report TR-496, NACA, 1935.

${ }^{17}$ H. Wagner. Über die Entstehung des dynamischen Auftriebes von Tragflügeln. ZAMM-Z Angew. Math. Me., 5(1):17-35, 1925.

${ }^{18} \mathrm{C}$. Wang and J. D. Eldredge. Low-order phenomenological modeling of leading-edge vortex formation. Theor. Comput. Fluid Dyn., 27(5):577-598, August 2013.

${ }^{19}$ D. Williams, W. Kerstens, S. Buntain, V. Quach, J. Pfeiffer, R. King, G. Tadmor, and T. Colonius. Closed-loop control of a wing in an unsteady flow. 48th AIAA Aerospace Sciences Meeting, January 2010, Orlando. AIAA 2010-0358, 2010. 
${ }^{20}$ J. C. Wu. Theory for Aerodynamic Force and Moment in Viscous Flows. AIAA Journal, 19(4):432-441, April 1981.

${ }^{21}$ X. Xia and K. Mohseni. Lift evaluation of a two-dimensional pitching flat plate. Phys. Fluids, 25:091901, 2013. 